03;04

\title{
Контракция микроволнового разряда в реакторе для газофазного осаждения алмаза
}

\author{
(ㄱ С.А. Богданов, А.М. Горбачев , Д.Б. Радищев, А.Л. Вихарев, М.А. Лобаев \\ Федеральный исследовательский центр Институт прикладной физики РАН, Нижний Новгород, Россия \\ ฯ E-mail: gorb@appl.sci-nnov.ru
}

Поступило в Редакцию 12 ноября 2018 г.

Обнаружено, что в смеси водород-метан в микроволновом плазменном реакторе для осаждения алмаза существует пороговое давление, начиная с которого происходит контракция микроволнового разряда. Приведены результаты измерений температуры газа и пространственных распределений интенсивности оптического излучения разряда. Обсуждается механизм контракции разряда.

DOI: 10.21883/PJTF.2019.03.47269.17585

В настоящее время химическое осаждение из газовой фазы (chemical vapor deposition, CVD) алмаза в реакторах на основе микроволнового разряда является хорошо освоенной технологией $[1,2]$, позволяющей получать поли- имонокристаллический CVD-алмаз высокого качества. B CVD-реакторе для достижения больших скоростей осаждения монокристаллического алмаза используются режимы работы, при которых обеспечивается высокое значение плотности микроволновой мощности, поглощаемой в плазме разряда [3,4]. Типичные значения плотности мощности составляют $50-200 \mathrm{~W} / \mathrm{cm}^{3}$, а в некоторых реакторах [3] достигаются даже более высокие значения 300-1000 W/ $\mathrm{cm}^{3}$. Плотность мощности в таких реакторах возрастает с увеличением давления [3,5]. Рост плотности мощности сопровождается увеличением концентрации атомарного водорода $[5,6]$, который играет ключевую роль в процессе CVD-синтеза алмаза [7]. Соответственно увеличение концентрации атомарного водорода позволяет значительно увеличить скорость роста алмаза [3,6]. Например, при давлении 300 Torr концентрация атомов водорода в объеме плазмы достигает $40 \%$ от полного числа молекул [6]. Возникает вопрос: до какого предела можно повышать давление газовой смеси в CVD-реакторе с целью увеличения концентрации атомов водорода?

В настоящей работе представлены результаты исследований разряда в микроволновом CVD-реакторе для осаждения алмаза в ранее неисследованном диапазоне давлений газа 300-500 Torr. Обнаружено, что в смеси водород-метан, которая обычно используется для синтеза алмаза, существует пороговое давление, начиная с которого происходит контракция микроволнового разряда. Приведены результаты измерений температуры газа и пространственного распределения интенсивности оптического излучения разряда. Обсуждается механизм контракции разряда.

Исследования проводились в плазмохимическом реакторе на основе цилиндрического резонатора (рис. 1,a). Резонатор 1 возбуждался коаксиальным волноводом 2 на моде $\mathrm{TM}_{013}$ на частоте $2.45 \mathrm{GHz}$. Область существования разряда 3 ограничивалась кварцевой колбой 4 и стенкой резонатора. Рабочая смесь газов подавалась в объем колбы, в котором давление газа поддерживалось постоянным за счет регулируемой скорости откачки. Разряд возникал в области максимальных значений электрического поля в резонаторе у поверхности подложки 5, на которую осаждалась алмазная пленка. Для наблюдения разряда в резонаторе имелись окна 6 , представляющие собой сетку из большого числа мелких (диаметром $2 \mathrm{~mm}$ ) часто расположенных отверстий. Для исследования пространственного распределения излучения разряда перед окном в резонаторе располагалась фокусирующая линза 7. В плоскости изображения разряда помещался вход световода 8 , через который оптическое излучение разряда поступало на вход спектрометра Horiba Jobin Yvon FHR-1000 с CCD-камерой Synapse CCD-2048 $\times 512$. Световод перемещался в плоскости изображения, при этом его ось всегда была направлена на центр линзы. Отметим, что окно 6 и центр линзы 7 располагались несколько выше поверхности подложки 5. С помощью известного отношения расстояний от оси резонатора и разряда до линзы и от линзы до входа световода перемещения световода пересчитывались в перемещение фокальной области по вертикальной оси разряда $Z$ и по перпендикулярной ей оси $R$ (рис. 1). При этом за начало отсчета $(z=0)$ было принято положение световода, при котором линия световод-центр линзы проходит через центр подложки. Разумеется, при такой схеме измерений (под небольшим углом к плоскости подложки) будет наблюдаться излучение и при $z<0$. Пространственное разрешение составляло около $2-3 \mathrm{~mm}$.

Эксперименты проводились как в чистом водороде, так и в смеси газов $\mathrm{H}_{2}+\mathrm{CH}_{4}$ при содержании метана $1 \%$. Поглощаемая в реакторе микроволновая мощность составляла $2.3 \mathrm{~kW}$.

Зависимость величины плотности мощности от давления для данного реактора была представлена нами в работе [5]. Плотность мощности менялась от $50 \mathrm{~W} / \mathrm{cm}^{3}$ при давлении 80 Torr до $500 \mathrm{~W} / \mathrm{cm}^{3}$ при давлении 350 Torr. 


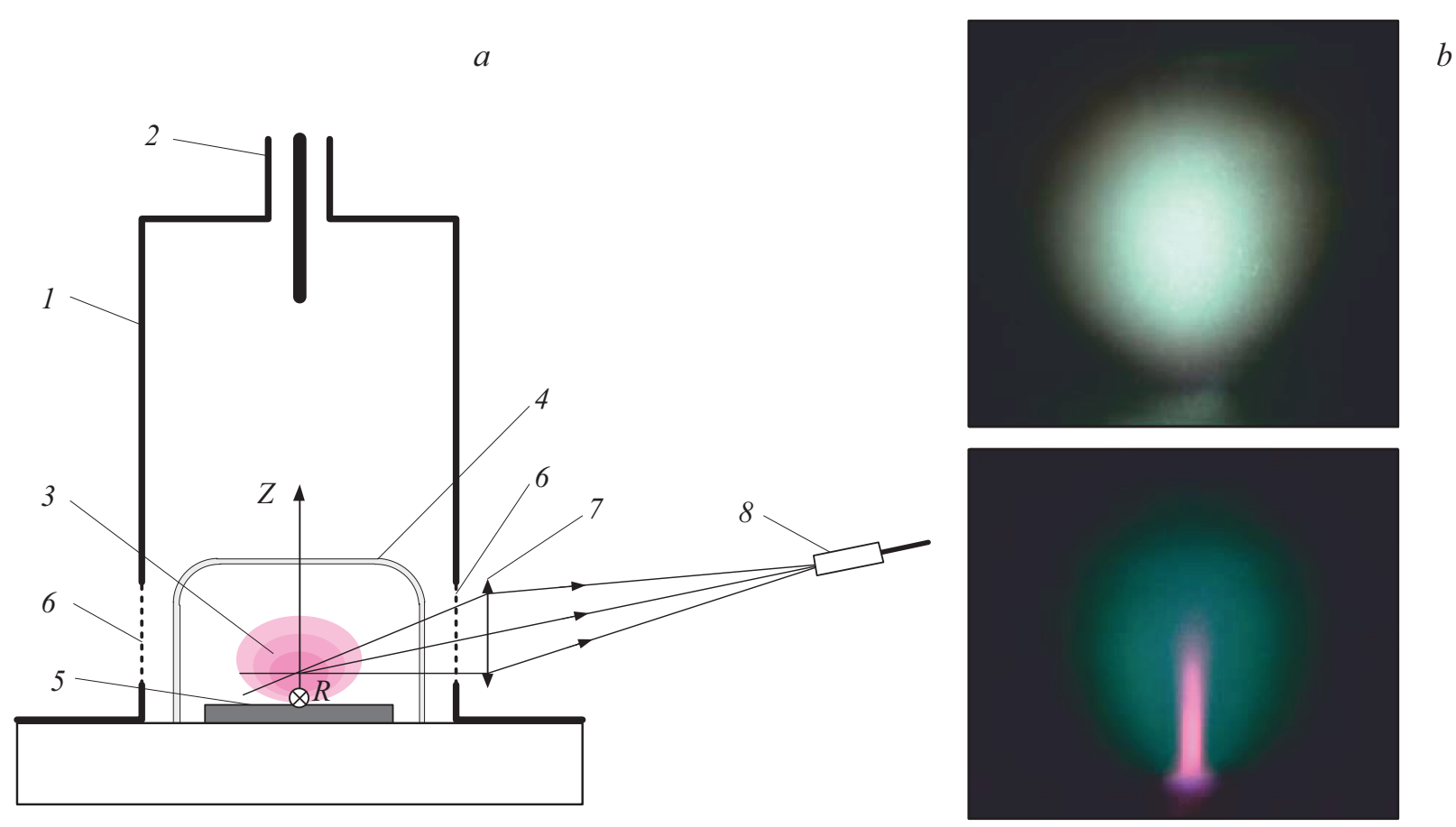

Рис. 1. $a$ - схема эксперимента: 1 - микроволновый цилиндрический резонатор, $2-$ коаксиальный волновод, 3 - газоразрядная плазма, 4 - кварцевая колба, 5 - подложка, 6 - окна в резонаторе, 7 - фокусирующая линза, $8-$ световод; $b-$ фотографии разряда: диффузная форма при давлении 350 Torr (вверху) и контрагированная при давлении 380 Torr (внизу).

Во всем диапазоне давлений как в чистом водороде, так и в смеси газов $\mathrm{H}_{2}+\mathrm{CH}_{4}$ наблюдаемая форма разряда имела вид эллипсоида, объем которого уменьшался с ростом давления. В настоящей работе продолжены исследования в области более высоких давлений и, соответственно, бо́льших значений плотности мощности. В чистом водороде поведение разряда не изменилось, с ростом давления форма разряда качественно не менялась, а объем плазмы уменьшался. Однако в смеси газов $\mathrm{H}_{2}+\mathrm{CH}_{4}$ при превышении давления 375 Torr форма разряда скачкообразно менялась, разряд переходил в контрагированную форму с ярким тонким цилиндрическим филаментом, вытянутым вдоль направления электрического поля (рис. $1, b)$. Такая форма разряда сохранялась при увеличении давления до 500 Torr (наибольшее рабочее давление в данном реакторе). При уменьшении давления газа наблюдался гистерезис, контрагированная форма переходила в однородную (диффузную) (рис. $1, b$ ) при снижении давления на 20-30 Torr от порога возникновения.

Для понимания причины контракции разряда в данном эксперименте нами были измерены пространственные распределения интенсивности излучения линии атомарного водорода $\mathrm{H} \alpha$ и молекулы $\mathrm{C}_{2}$ (переход $d^{3} \Pi_{g} \rightarrow a^{3} \Pi_{u}$ около $516 \mathrm{~nm}$ ) (рис. 2 и 3). Хорошо видно, что при контракции имеет место резкое изменение объема плазмы, из которого происходит излучение атомов водорода. При этом, как видно из фотографии разряда (рис. $1, b)$ и радиального распределения интенсивности (рис. $2, b$ ), размер области, в которой имеется атомарный водо- род, вблизи подложки составляет всего несколько миллиметров. Очевидно, что в таком режиме осаждение CVD-алмаза даже на небольшие монокристаллические подложки (например, $3 \times 3 \mathrm{~mm}$ ) может быть сильно неоднородным или даже невозможным. На рис. 2 приведены нормализованные значения интенсивностей, но абсолютная величина интенсивности излучения (в максимуме ее значения) при контракции возрастает более чем в 10 раз.

Распределение интенсивности излучения молекулы $\mathrm{C}_{2}$ в контрагированной форме разряда шире, чем распределение интенсивности излучения атомарного водорода. Это хорошо видно и на фотографии разряда (рис. $1, b$ ), где яркий филамент на оси разряда имеет сиреневый оттенок (излучение молекулярного и атомарного водорода), а окружающий его ореол зеленого цвета (излучение молекулы $\mathrm{C}_{2}$ ). Возбуждение атомов водорода с высокой энергией возбуждения (энергия третьего уровня, соответствующего излучению линии $\mathrm{H} \alpha$, равна $12.1 \mathrm{eV}$ ) происходит посредством электронного удара и наблюдается в области, где велика концентрация электронов и атомов водорода. Излучение молекулы $\mathrm{C}_{2}$ наблюдается из более широкой области, что может быть объяснено тем, что в возбуждении уровня $d^{3} \Pi_{g}$ (энергия $2.41 \mathrm{eV}$ ) большую роль играют процессы без участия электронов, столкновения молекул и/или хемолюминесценция $[8,9]$.

Как известно [10], для контракции разряда необходимо выполнение двух условий. Во-первых, зависимость частоты ионизации нейтральных частиц от концентра- 

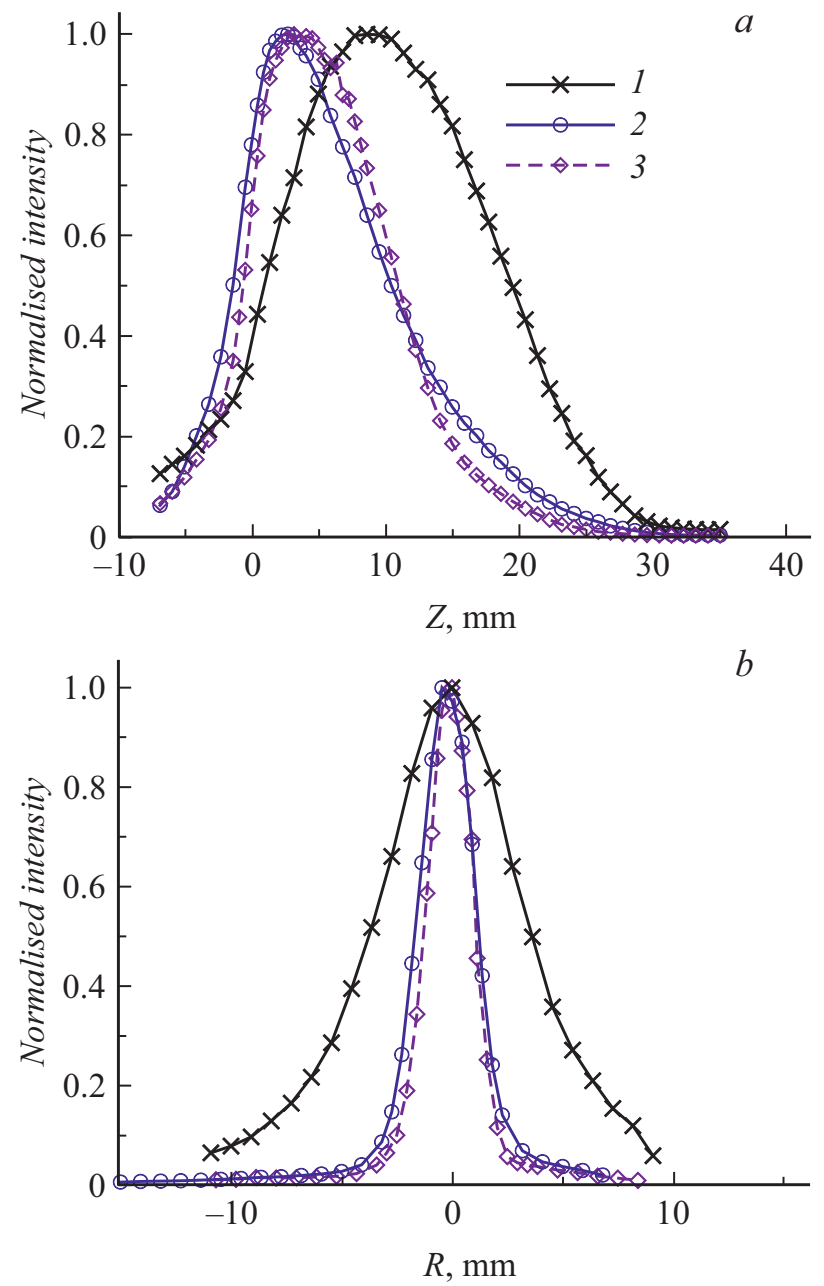

Рис. 2. Распределения интенсивности излучения линии атомарного водорода $\mathrm{H} \alpha . a-$ вдоль оси разряда $Z, b-$ вдоль оси $R .1$ - однородный разряд при давлении 373 Torr, 2 - контрагированный разряд при давлении 375 Torr, 3 контрагированный разряд при давлении 500 Torr.

ции электронов должна быть нелинейной, а во-вторых, гибель электронов должна иметь объемный характер. Хорошо известен механизм образования нитевидной структуры в микроволновом разряде в результате развития ионизационно-перегревной неустойчивости [11], природа которой связана с уменьшением плотности газа из-за его нагрева и увеличением энергии электронов и, следовательно, частоты ионизации за счет роста величины приведенного электрического поля $E / N(E-$ среднеквадратичная напряженность электрического поля, $N-$ концентрация молекул). По вращательной структуре излучения перехода $d^{3} \Pi_{g} \rightarrow a^{3} \Pi_{u}$ молекулы $\mathrm{C}_{2}$ [12] была определена температура газа, распределение которой также представлено на рис. 3. Из рисунка видно, что нагретая область заметно превышает размеры филамента, в области которого наблюдается лишь незначительное увеличение температуры. Поэтому в данном случае контракцию разряда нельзя связать с развитием ионизационно-перегревной неустойчивости.

Отметим, что контракция разряда происходит в смеси $\mathrm{H}_{2}+\mathrm{CH}_{4}$, в чистом водороде она не наблюдалась. Добавка метана к водороду приводит к смене иона с $\mathrm{H}_{3}^{+}$на углеродсодержащие ионы, которые имеют значительно больший коэффициент рекомбинации и меньшую подвижность $[13,14]$. Увеличение рекомбинации и уменьшение диффузии обеспечивают объемные потери электронов. Оценки показывают, что диффузионная длина потерь за счет рекомбинации $\sqrt{D_{a} /\left(\alpha N_{e}\right)}$ (где $D_{a}-$ коэффициент диффузии, $\alpha-$ коэффициент рекомбинации, $N_{e}-$ концентрация электронов) составляет порядка 1-2 mm. Нелинейная зависимость частоты ионизации нейтралов возникает из-за известного эффекта, состоящего в том, что при диссоциации молекулярного газа происходит увеличение энергии электронов [11]. Таким образом, увеличение концентрации электронов приводит к росту степени диссоциации молекулярного водорода, что ведет к увеличению температуры электронов и константы ионизации электронным ударом. Как показывают актинометрические измерения, степень
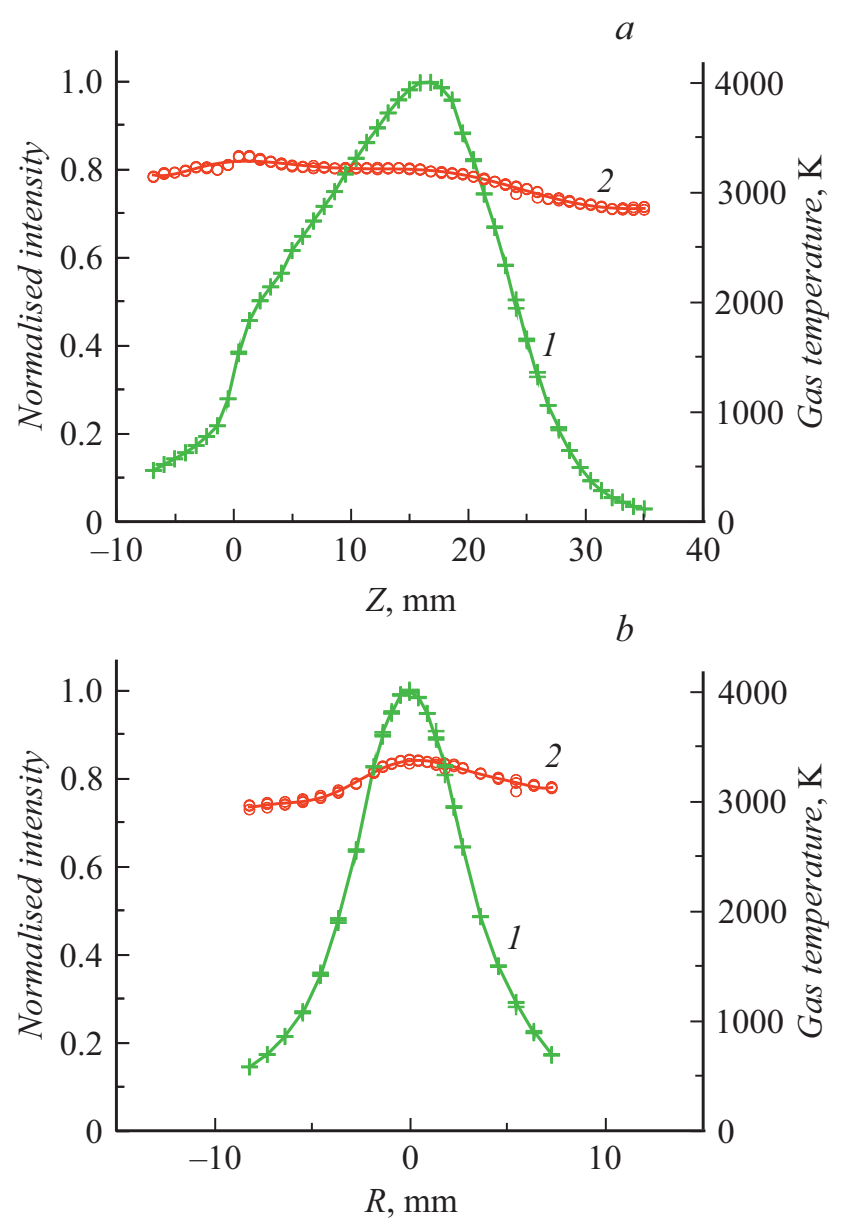

Рис. 3. Распределения интенсивности излучения молекулы $\mathrm{C}_{2}$ (переход $d^{3} \Pi_{g} \rightarrow a^{3} \Pi_{u}$ около $516 \mathrm{~nm}$ ) (1) и температуры газа (2) в контрагированной форме разряда при давлении 375 Torr. $a$ - вдоль оси разряда $Z, b-$ вдоль оси $R$. 
диссоциации водорода увеличивается с ростом давления газа $[5,6]$.

В работе впервые показано, что микроволновый разряд в смеси газов $\mathrm{H}_{2}+\mathrm{CH}_{4}$ в CVD-реакторе для осаждения алмаза при достижении некоторого порогового давления переходит в контрагированную форму. Образование контрагированной формы может быть объяснено объемными потерями электронов за счет быстрой рекомбинации углеродсодержащих ионов и нелинейным механизмом роста концентрации электронов из-за увеличения их энергии с повышением степени диссоциации водорода. Контракция разряда при высоких давлениях препятствует достижению высокой плотности микроволновой мощности, поглощаемой в плазме в однородной форме разряда. Таким образом, обнаруженная контракция разряда в водород-метановой газовой смеси накладывает ограничения на режимы работы CVD-реакторов для осаждения алмаза.

Работа выполнена в рамках государственного задания ИПФ РАН (проект № 0035-2014-0002).

\section{Список литературы}

[1] Balmer R.S., Brandon J.R., Clewes S.L., Dhillon H.K., Dodson J.M., Friel I., Inglis P.N., Madgwick T.D., Markham M.L., Mollart T.P. // J. Phys.: Condens. Matter. 2009. V. 21. N 36. P. 364221.

DOI: $10.1088 / 0953-8984 / 21 / 36 / 364221$

[2] Power electronics device applications of diamond semiconductors / Eds S. Koizumi, H. Umezawa, J. Pernot, M. Suzuki. Woodhead Publ., 2018. 466 p.

[3] Gu Y., Lu J., Grotjohn T., Schuelke T., Asmussen J. // Diamond Relat. Mater. 2012. V. 24. P. 210-214. DOI: 10.1016/j.diamond.2012.01.026

[4] Muchnikov A.B., Vikharev A.L., Gorbachev A.M., Radishev D.B., Blank V.D., Terentiev S.A. // Diamond Relat. Mater. 2010. V. 19. N 5-6. P. 432-436.

DOI: 10.1016/j.diamond.2009.11.012

[5] Lobaev M.A., Bogdanov S.A., Radishev D.B., Vikharev A.L., Gorbachev A.M. // Diamond Relat. Mater. 2016. V. 66. P. 177182. DOI: $10.1016 /$ j.diamond.2016.05.004

[6] Derkaoui N., Rond C., Hassouni K., Gicquel A. // J. Appl. Phys. 2014. V. 115. N 23. P. 233301. DOI: $10.1063 / 1.4883955$

[7] Goodwin D.G. // J. Appl. Phys. 1993. V. 74. N 11. P. 68886894. DOI: $10.1063 / 1.355063$

[8] Gaydon A.G., Wolfhard H.G. // Proc. R. Soc. Lond. A. 1950. V. 201. P. 561-569.

[9] Duten X., Rousseau A., Gicquel A., Leprince P. // J. Appl. Phys. 1999. V. 86. N 9. P. 5299-5301. DOI: $10.1063 / 1.371515$

[10] Райзер Ю.П. Физика газового разряда. М.: Наука, 1987. 592 c. [Raizer Y.P. Gas discharge physics. Berlin-Heidelberg: Springer-Verlag, 1991. 449 p.].

[11] FridmanAA., Kennedy L.A. Plasma physics and engineering. Taylor \& Francis Routledge, 2004. 853 p.

[12] Vikharev A.L., Gorbachev A.M., Radishev D.B. // J. Phys. D: Appl. Phys. 2019. V. 52. N 1. P. 014001. DOI: $10.1088 / 1361-6463 / a a e 3 a 3$

[13] Ma J., Ashfold M.N.R., Mankelevich Y.A. // J. Appl. Phys. 2009. V. 105. N 4. P. 043302. DOI: $10.1063 / 1.3078032$

[14] Лебедев Ю.А., Эпштейн И.Л. // Теплофизика высоких температур. 1998. Т. 36. № 4. С. 534-540. 